\title{
INVESTIGACIONES
}

\section{Evaluación de la alfabetización probabilística del profesorado en formación y en activo}

\author{
Evaluation of probabilistic literacy of active and in-service teachers
}

\author{
Francisco Rodríguez-Alveal ${ }^{a}$, Danilo Díaz-Levicoy ${ }^{b}$ Claudia Vásquez Ortiz \\ ${ }^{\text {a }}$ Departamento de Ciencias de la Educación, Universidad del Bío-Bío. \\ Correo electrónico: frodriguez@ubiobio.cl \\ b Universidad de Granada. \\ Correo electrónico: dddiaz01@ @otmail.com. \\ ' Pontificia Universidad Católica de Chile, Campus Villarrica. \\ Correo electrónico: cavasque@uc.cl.
}

\begin{abstract}
RESUMEN
Este trabajo tiene por objetivo comparar el conocimiento sobre aspectos de alfabetización probabilística que presentan profesores en activo y en formación. Para recoger los datos se aplicó, un instrumento construido para tal finalidad, cuya fiabilidad fue cuantificada mediante el Coeficiente alfa de Cronbach, entregando un valor $(\mathrm{a}=0,78)$ adecuado para su propósito. La muestra estuvo constituida por 30 profesores en formación y 15 en activo, la que se obtuvo mediante un muestreo no probabilístico del tipo intencionado. En general, los resultados muestran que los profesores en activo presentan porcentajes de logro menos descendidos que los profesores en formación en relación a identificar secuencias aleatorias, reglas probabilísticas. En cambio, los profesores en formación presentan porcentajes de logro superiores en cálculo de probabilidad en enunciados de tipo textual. Además, ambos grupos entregan argumentaciones poco plausibles, evidenciando una formación de carácter procedimental sobre la conceptual que permitiría dar respuesta a la habilidad argumentativa.
\end{abstract}

Palabras clave: Comprensión, Probabilidad, Formación Docente, Educación Secundaria.

\begin{abstract}
The objective of this paper is to compare the knowledge of probabilistic literacy aspects possessed by active and in-service teachers. To collect the data, an instrument was constructed and applied whose reliability was quantified by Cronbach's alpha coefficient, which delivered a value $(\alpha=0.78)$ that is adequate for this purpose. The sample consisted of 30 teachers in training and 15 active teachers, and it was obtained by a non-probabilistic sampling of the intended type. In general, the results show that active teachers have higher percentages of achievement than teachers in training in relation to identifying random sequences and probabilistic rules. On the other hand, teachers in training have higher percentages of achievement in the calculation of probability in textual statements. In addition, both groups give little plausible argumentation, evincing a training more procedural than conceptual, which would allow an answer to be given regarding argumentative ability.
\end{abstract}

Keywords: understanding, probability, teacher training, Secondary Education. 


\section{INTRODUCCIÓN}

En Chile, en los últimos años, se ha fortalecido y reformulado las directrices curriculares, en particular en el área de matemáticas, introduciendo el eje de Datos y Probabilidades desde la Educación Primaria (Ministerio de Educación 2012a). En ellas se establecen los contenidos y habilidades que deben trabajar los estudiantes para lograr la compresión de conceptos relacionados al razonamiento matemático al enfrenarse a la resolución de problemas estadísticos.

Entre los objetos estadísticos considerados en las directrices curriculares se encuentra el de probabilidad, que se introduce tempranamente en varios países a nivel mundial. Chile no es ajeno a esta tendencia, que es una consecuencia directa de la globalización y de la modernidad, así como, de la gran cantidad de información de tipo cultural, política, económica, educativa o social en la cual la incertidumbre se encuentra presente ( $\mathrm{Gal} 2005$ ). De ahí la necesidad de contar con ciudadanos críticos cuyos conocimientos les permitan interpretar, seleccionar y comunicar información, en definitiva, ciudadanos "capaces de hacer frente a una amplia gama de situaciones del mundo real que implican la interpretación o la generación de mensajes probabilísticos, así como la toma de decisiones" (Gal 2005).

Por tanto, es interesante, indagar acerca de los elementos cognitivos y disposicionales que presentan profesores de matemática en formación y en activo en relación con la alfabetización probabilística, como una forma de dar respuesta a los estándares que guían la formación que establece el Ministerio de Educación. Por un lado, profesores de primaria, tras finalizar su formación deben:

(...) conoce $[\mathrm{r}]$ en profundidad los contenidos del eje de Datos y Probabilidades del currículo escolar respecto de Probabilidades. Domina[r] conceptos básicos de este tema que le permiten analizar y describir fenómenos aleatorios, cuantificando y representando la probabilidad de ocurrencia de eventos, y presentar el tema de Probabilidades como herramienta matemática que sirve para modelar el azar y que aporta a la toma de decisiones. Es capaz de conducir el aprendizaje de los estudiantes en la introducción de conceptos relativos a probabilidades, utilizando para esto situaciones lúdicas y cotidianas que ilustren como cuantificar el azar, considerando las dificultades que esto conlleva en cada nivel (Ministerio de Educación, 2012b).

Del mismo modo, los que egresan como profesores de Educación Secundaria, deben:

(...) aplica[r] técnicas de conteo y conoce[r] la concepción de probabilidad en el caso equiprobable (modelo de Laplace) y también en el caso no equiprobable. Comprende[r] el significado empírico de las probabilidades y es capaz de ilustrarlo con medios concretos y con simulaciones. Conoce $[\mathrm{r}]$ y aplica[r] las probabilidades condicionales y comprende[r] la relación de éstas con la independencia de eventos aleatorios, integrando estos conceptos en los teoremas de probabilidades totales y de Bayes (Ministerio de Educación 2012c).

Estos estándares reflejan la importancia de contar con profesores con una sólida formación en probabilidad. ¿Por qué en este estudio nos centramos en los profesores de Educación Secundaria?, Porque de acuerdo con las demandas actuales es muy frecuente 
que los profesores de Educación Secundaria deban impartir clases en los cursos finales de Educación Primaria, dado que estos han recibido una formación inicial docente de carácter más específico que les permitirá, supuestamente, abordar de mejor manera los contenidos que se encuentran al finalizar la Educación Primaria.

Así, en el caso del estudio de la probabilidad en Educación Primaria, este se inicia a partir del trabajo con datos, tablas y gráficos, para luego seguir con el desarrollo de experimentos aleatorios que darán paso a describir la probabilidad a partir del grado de posibilidad de ocurrencia de sucesos provenientes de situaciones cotidianas diversas. En niveles posteriores se prosigue con la comparación de probabilidades, pero sin calcularlas, a partir de conjeturas acerca de las tendencias de resultados obtenidos de un mismo experimento, que se pretende que los estudiantes lleguen a estimar la probabilidad teórica de un evento. Para que en los últimos años de Educación Primaria procedan con el estudio de la probabilidad asociada a frecuencias relativas de ocurrencia de eventos que se estabilizan acercándose cada vez más a la probabilidad de ocurrencia del suceso, para así dar paso a distintos tipos de representar numéricamente dicha probabilidad. Finalizando, con el principio combinatorio multiplicativo y sus representaciones como una herramienta para determinar el espacio muestral de un suceso y para el cálculo de probabilidad.

En coherencia con lo anterior, como menciona Even y Ball (Even y Ball 2009), este tipo de estudios cobra especial relevancia dado que:

- Los profesores cumplen un rol fundamental en el proceso de enseñanza y aprendizaje de la matemática en los estudiantes de los diferentes niveles de formación.

- La formación que reciben los futuros profesores es un elemento clave para la construcción de un sistema efectivo en la enseñanza de la matemática.

- La formación de profesores en general, y de matemática en particular, es una problemática que presenta grandes desafíos, tanto para las instituciones formadores para la sociedad.

Para Liljedahl las investigaciones que se realizan en torno al conocimiento sobre profesores, en formación y/o en activo, se relacionan al conocimiento del contenido, conocimiento pedagógico y conocimiento didáctico (Liljedahl 2009). En Educación Matemática, como mencionan Inzunsa y Guzmán "el conocimiento de contenido contempla conceptos matemáticos, uso de técnicas matemáticas, razonamiento matemático, demostraciones, entre otros; el conocimiento pedagógico contempla principios generales de educación tales como teorías de aprendizaje y aspectos éticos de la educación; mientras que el conocimiento didáctico es el conocimiento relacionado con las condiciones y las maneras de enseñar y aprender matemáticas" (Inzunsa y Guzmán 2011).

De acuerdo a estas consideraciones es que en este trabajo se pretende indagar sobre la alfabetización probabilística de profesores de matemática en formación y en activo, de acuerdo con los estándares orientadores para carreras de pedagogía en Educación Secundaria (Ministerio de Educación 2012c). Con este propósito en mente, el presente trabajo se ha estructurado en varios apartados: en la Sección 2 se exponen los elementos teóricos y antecedentes que han sido empleados, en la Sección 3 se recogen la metodología de este estudio (característica de la muestra e instrumento de evaluación), en la Sección 4 se presentan los principales resultados obtenidos y, por último, en la Sección 5 se presentan las conclusiones más destacadas del estudio. 


\section{FUNDAMENTACIÓN}

En este apartado se describen los fundamentos teóricos relacionados a la alfabetización probabilística y los antecedentes sobre el conocimiento sobre probabilidad de profesores de Educación Primaria y Secundaria en diferentes niveles formativos.

\subsection{ALFABETIZACIÓN PROBABILÍSTICA}

Las demandas de la sociedad varían muy rápidamente, cambiando al mismo tiempo lo que los niños necesitan aprender en su formación obligatoria. Lo anterior, trae consigo la necesidad de contar con ciudadanos críticos, capaces de interpretar adecuadamente la gran avalancha de datos a las cuales se ven y verán enfrentados a diario, así como enfrentarse a diferentes situaciones de incertidumbre. En el sistema escolar, la estadística se declara usualmente en la asignatura de matemática (Batanero 2001; Del Pino y Estrella 2012). Sin embargo, ellas operan en ambientes o mundos diferentes, con lenguajes y concepciones propias. La matemática trata principalmente con lo determinístico y deductivo; y la estadística con lo estocástico y lo inductivo, donde la variación y el contexto real son esenciales en su enseñanza (Estrella, Olfos y Mena-Lorca 2015). Producto de lo anterior, es necesario que los profesores en formación y en activo, a nivel de Educación Primaria, Secundaria y Universitaria, consideren que cada contenido matemático y estadístico posee complejidades diferentes que demandan una forma distinta de enseñanza (Ben-Zvi y Garfield 2004; Franklin y Garfield 2006).

En coherencia con lo anterior, las vigentes directrices curriculares del Ministerio de Educación chileno, por medio del eje de Datos y Probabilidades, demandan que los profesores consigan alfabetizar estadísticamente y probabilísticamente a sus estudiantes. Tradicionalmente el término alfabetización ha sido asociado a la lectura y escritura en la lengua materna, concepto que se ha extrapolado hacia otras disciplinas. En este contexto, para Gimeno "la alfabetización eficaz supone poner a los sujetos a las puertas del poder que implica la posesión del conocimiento a través del dominio del lenguaje" (Gimeno 1999).

Según Gal, en estadística, el término alfabetización hace referencia a la capacidad que deben tener los ciudadanos para comprender información de tipo estadística a la que acceden en la vida cotidiana, y que lo define como la unión de las siguientes competencias:

a) Interpretar y evaluar críticamente la información estadística, los argumentos apoyados en datos o los fenómenos estocásticos que las personas pueden encontrar en diversos contextos, incluyendo los medios de comunicación, pero no limitándose a ellos; y b) discutir o comunicar sus opiniones respecto a tales informaciones estadísticas cuando sea relevante" (Gal 2002)

De modo similar, el término alfabetización probabilista (cultura probabilista o cultura básica en probabilidad) hace referencia a la importancia del conocimiento probabilístico elemental en los ciudadanos y para su interacción en la vida cotidiana (Batanero 2005, 2006; Gal 2005; Sánchez 2009). Gal, por su parte, menciona la necesidad de una adecuada cultura estadística, ya que: (1) la probabilidad es una parte importante de la estadística, que sirve como base para estudiar temas más avanzados; (2) ayuda a preparar para la 
vida cotidiana, porque se identifican eventos aleatorios y fenómenos de azar, en el ámbito personal y profesional (Gal 2005).

Desde esta perspectiva, Gal propone un modelo para el desarrollo de la alfabetización probabilística a partir de ciertos componentes básicos (Tabla 1 y 2). En ellas vemos que el lenguaje cotidiano e informal, vinculado al significado intuitivo de la probabilidad, constituye un elemento de base para construir una conexión con el lenguaje probabilístico, que permitirá que los estudiantes comiencen a utilizar un lenguaje preciso y especializado para expresar de forma cualitativa la probabilidad de ocurrencia de un determinado suceso (Gal 2002, 2005).

Tabla 1. Elementos disposicionales de la alfabetización probabilística (Gal 2002)

\begin{tabular}{|l|l|}
\hline Elementos disposicionales & \multicolumn{1}{|c|}{ Actitudes } \\
\hline Postura crítica. & $\begin{array}{l}\text { Los mensajes cuantitativos que pueden ser engañosos, unilaterales, } \\
\text { sesgados o incompletos (ya sea intencionalmente o no) deberían } \\
\text { generar una actitud de cuestionamiento. } \\
\text { Es, pues, necesario aprender progresivamente a invocar de forma } \\
\text { espontánea la lista de preguntas que generan incertidumbre frente a } \\
\text { argumentos que pretenden basarse en datos, informes de resultados, } \\
\text { conclusiones de encuestas u otras investigaciones empíricas. }\end{array}$ \\
\hline Creencias y actitudes. & $\begin{array}{l}\text { Se distinguen tres constructos distintos dentro del dominio afectivo } \\
\text { en educación matemática: emociones, actitudes y creencias. } \\
\text { Es, pues, necesario desarrollar progresivamente una visión positiva } \\
\text { de sí mismo como individuo capaz de realizar razonamientos } \\
\text { probabilísticos en situaciones de incertidumbre que sean relevantes, } \\
\text { más que partir de datos anecdóticos o de experiencias personales. }\end{array}$ \\
\hline $\begin{array}{l}\text { Los sentimientos personales } \\
\text { en relación a la incertidumbre } \\
\text { y el riesgo (por ejemplo, la } \\
\text { aversión al riesgo). }\end{array}$ & $\begin{array}{l}\text { El grado de incertidumbre o previsibilidad experimentado puede ser } \\
\text { la base de la propia percepción y capacidad para evaluar el riesgo } \\
\text { asociado con los eventos o resultados de relevancia para la vida. }\end{array}$ \\
\hline
\end{tabular}


Tabla 2. Elementos cognitivos de la alfabetización probabilística (Gal 2005)

\begin{tabular}{|c|c|}
\hline Elementos cognitivos & Conocimientos \\
\hline $\begin{array}{l}\text { Grandes ideas de probabili- } \\
\text { dad: Variación, aleatoriedad, } \\
\text { independencia, predicción/in- } \\
\text { certidumbre. }\end{array}$ & $\begin{array}{l}\text { La alfabetización probabilística es una construcción dinámica y } \\
\text { relativa. } \\
\text { La aleatoriedad es una construcción resbaladiza que ha sido } \\
\text { debatida por muchos estadistas (un posible punto de vista es que el } \\
\text { azar es una característica de un resultado). } \\
\text { La independencia implica que los eventos son inconexos y un } \\
\text { evento no se puede predecir de otro. } \\
\text { La predicción y la incertidumbre se relacionan con el estado de } \\
\text { nuestro conocimiento general acerca de la probabilidad de un } \\
\text { determinado evento. }\end{array}$ \\
\hline $\begin{array}{l}\text { Asignación de probabili- } \\
\text { dades: formas de encontrar o } \\
\text { estimar la probabilidad de } \\
\text { ocurrencia de un evento. }\end{array}$ & $\begin{array}{l}\text { Para calcular las probabilidades, los alumnos deben estar } \\
\text { familiarizados con la manera de encontrar la probabilidad de } \\
\text { eventos, con el fin de entender los estados probabilísticos realizados } \\
\text { por otros, o para generar estimaciones sobre la probabilidad de } \\
\text { eventos y comunicarse con los demás acerca de ellos. Aquí es donde } \\
\text { los puntos de vista de la probabilidad clásica, frecuentista y } \\
\text { subjetiva son útiles. }\end{array}$ \\
\hline $\begin{array}{l}\text { Lenguaje: los términos y los } \\
\text { métodos utilizados para co- } \\
\text { municar el azar }\end{array}$ & $\begin{array}{l}\text { Los alumnos deben entender el "lenguaje probabilístico", es decir, } \\
\text { las diversas formas que se utilizan para representar y comunicar } \\
\text { acerca del azar y la probabilidad. } \\
\text { La probabilidad de eventos se puede representar cuantitativamente } \\
\text { por múltiples sistemas, como en una escala de } 0-1 \text {, fracciones (por } \\
\text { ejemplo, 50/50), porcentajes, proporciones, etc., así como } \\
\text { gráficamente. Por lo tanto, una expectativa básica es que los } \\
\text { alumnos entiendan la intercambiabilidad de diferentes } \\
\text { representaciones y se sientan cómodos moviéndose entre ellas. }\end{array}$ \\
\hline $\begin{array}{l}\text { Contexto: La comprensión } \\
\text { del papel y las implicaciones } \\
\text { de los problemas probabilísti- } \\
\text { cos y mensajes en diferentes } \\
\text { contextos y en el discurso per- } \\
\text { sonal y público. }\end{array}$ & $\begin{array}{l}\text { Los conocimientos relativos al contexto son necesarios tanto desde } \\
\text { el punto de vista funcional como educativo. La comprensión de que } \\
\text { el azar y la aleatoriedad no afectarán a los acontecimientos y } \\
\text { procesos del mundo real permite a las personas prever que ciertos } \\
\text { eventos serán más predecibles, mientras que otros no tanto. }\end{array}$ \\
\hline $\begin{array}{l}\text { Preguntas críticas: Cuestiones } \\
\text { para reflexionar cuando se tra- } \\
\text { ta de probabilidades. }\end{array}$ & $\begin{array}{l}\text { Los alumnos deben saber qué preguntas críticas realizar cuando se } \\
\text { encuentran con una declaración de probabilidad o certeza, o cuando } \\
\text { tienen que generar una estimación probabilística. } \\
\text { Las preguntas deberían referirse a } 5 \text { elementos: el contexto (¿en qué } \\
\text { medida implica aleatoriedad?); la fuente (¿quién hace una demanda } \\
\text { probabilística?); el proceso (¿qué tipo de análisis se usa?); el } \\
\text { significado del mensaje (¿qué indica la afirmación probabilística?); } \\
\text { y la interpretación reflexiva (¿qué cuestiona el mensaje y cómo se } \\
\text { interpreta? }\end{array}$ \\
\hline
\end{tabular}


En relación con los componentes de la alfabetización probabilística expuestos, Gal aclara que, a pesar de presentarlos por separado, todos ellos interactúan entre sí de manera compleja durante el aprendizaje real. Esto significa que una instrucción que se centre sólo en uno o dos de los elementos no será suficiente para desarrollar un comportamiento alfabetizado de probabilidad.

La incorporación de estos contenidos exige un buen desarrollo profesional de los futuros profesores y de los profesores en ejercicio, el cual incluye tres tipos de saberes docentes (Alsina 2012):

- Los saberes disciplinares: corresponden al propio campo de conocimiento.

- Los saberes pedagógicos: incluyen a los saberes curriculares, y se centran en cómo llevar a cabo la práctica educativa (conocimientos didácticos).

- Los saberes experienciales: los desarrollados por los maestros en su práctica educativa, y que deberían ser la base para reflexionar sobre la propia práctica y mejorarla.

Sin embargo, las investigaciones al respecto aún son escasas y evidencian "la necesidad de desarrollar investigaciones vinculadas a la naturaleza semiótica de la enseñanza y aprendizaje de la probabilidad" (Nilsson y Li 2015), que proporcionen herramientas al profesorado que les permitan implementar de manera idónea las nuevas directrices curriculares, sobre todo si consideramos que gran parte del profesorado en ejercicio no ha recibido formación al respecto. Producto de esta falta de preparación, en muchas ocasiones, la enseñanza de la probabilidad se omite, ya que es considerada un tema de menor importancia para la formación de los estudiantes (Serradó, Azcárate y Cardeñoso 2006), y cuando se realiza se focaliza, principalmente, en la enseñanza de fórmulas, dejando de lado la experimentación con fenómenos aleatorios y la resolución de problemas (Batanero, Ortiz, Serrano 2007). Por otra parte, los documentos curriculares y los libros de texto, que en muchas ocasiones constituyen un apoyo para la labor del profesor, en el caso de la probabilidad, no ofrecen el apoyo suficiente, pues éstos en su mayoría presentan una visión sesgada o incompleta de la probabilidad.

Por consiguiente es necesario otorgar formación específica al profesorado que les permita implementar exitosamente las actuales directrices curriculares, más aún si consideramos que la probabilidad es un concepto complejo, siendo fundamental que los profesores cuenten con los saberes docentes que les permitan orientar el proceso de enseñanza de una manera progresiva a partir de las ideas intuitivas de sus alumnos sobre el azar y probabilidad, para luego de manera gradual e ir construyendo poco a poco el concepto de probabilidad, pues tal y como dijo Laplace: "el aprendizaje de la probabilidad nos ayuda a evitar ilusiones en la toma de decisiones y por ello no hay ciencia más digna de nuestro estudio ni más útil para que se incluya en el sistema público de educación" (Laplace 1986).

\subsection{CONOCIMIENTO DEL PROFESORADO SOBRE PROBABILIDAD}

Gran parte de las actividades de enseñanza que realizan los profesores dependen directamente de su conocimiento matemático en relación al tema (Ball, Lubienski y Mewborn 2001). Por lo que resulta de gran importancia que los profesores cuenten con un 
sólido conocimiento de la probabilidad. Las investigaciones al respecto son muy escasas y por lo general se centran en profesores en formación; y se centran principalmente en determinar si los futuros profesores cuentan o no con los conocimientos suficientes para llevar a cabo una enseñanza idónea de la probabilidad. De ahí que este estudio constituye un aporte al campo de la formación del profesorado, pues se centra específicamente en el nivel de alfabetización probabilística que poseen profesores de matemática en formación y en activo.

Una de las primeras investigaciones en torno a las concepciones y el conocimiento probabilístico de futuros profesores de Educación Primaria fue realizada por Azcárate quien detectó concepciones erróneas y dificultades en relación a la noción de probabilidad; además de una baja comprensión de la noción de aleatoriedad y por ende en la comprensión del conocimiento probabilístico en una muestra de 57 profesores en formación. Esto se debía a que su razonamiento en relación a la noción de probabilidad, era más bien en base a un conocimiento de tipo cotidiano que en un conocimiento formal. Por otra parte, se detectaron serias dificultades en relación a la idea de juego equitativo, puesto que los futuros profesores, en su mayoría, no fueron capaces de diferenciar entre juego equitativo y no equitativo. Asimismo, se detectó la carencia de esquemas combinatorios y de instrumentos elementales para la asignación de probabilidades (Azcárate 1995).

Begg y Edward realizan entrevistas y aplican un cuestionario, a un grupo de 22 profesores australianos de primaria, quienes deben dar respuesta a tres situaciones relacionadas con ideas básicas de aleatoriedad, sucesos equiprobables e independencia. En base a las respuestas se detectó una escasa comprensión de la probabilidad y de las nociones que subyacen a ella, ya que alrededor de dos tercios de los profesores a quienes aplicaron el cuestionario, lograron dar respuesta satisfactoria a las situaciones planteadas. Además, fue posible evidenciar tres aspectos centrales, primero, que empleaban la heurística de la representatividad (Tversky y Kahneman 1982), es decir, una confianza excesiva en el comportamiento de las pequeñas muestras, llevándolos a generalizar erróneamente para la población, segundo, la presencia de la creencia que todos los sucesos aleatorios son equiprobables (Lecoutre 1992), y por último, dificultades para interpretar un enunciado de probabilidad de manera no probabilística (Konold 1991). Sin embargo, este grupo de profesores manifestó estar más preocupados de conseguir ideas y actividades para enseñar en el aula, que de mejorar su propio conocimiento en relación a la probabilidad y su enseñanza. Es importante señalar que este tipo de dificultades y concepciones erróneas no solo se encuentran presentes en los profesores de primaria, sino también en los de secundaria y sobre todo en lo que se refiere a la comprensión de la probabilidad condicional (Begg y Edward 1999).

Batanero, Godino y Cañizares evaluaron la presencia de sesgos en el razonamiento probabilístico en una muestra de 132 futuros profesores, obteniendo como resultado que un $60 \%$ de los profesores en formación, a los que se aplicó el cuestionario, razonaban según la heurística de la representatividad (Tversky y Kahneman 1982). Otro 60\% de la muestra presentó el sesgo de equiprobabilidad (Lecoutre 1992; Lecoutre y Durand 1988), mientras que un $23 \%$ de los futuros profesores presentó un "outcome approach" dificultad para interpretar un enunciado probabilístico en forma no probabilística (Konold 1991). Estos sesgos, así como el razonamiento probabilístico, fueron reducidos y mejorados al utilizar metodologías de enseñanza basadas en la simulación, con dispositivos manipulativos, tablas de números aleatorios y utilización de software, como recurso didáctico que ayudó a superar las dificultades y concepciones erróneas (Batanero, Godino y Cañizares 2005). 
Estrada y Díaz analizan los conocimientos probabilísticos de 65 profesores en formación de Educación Primaria sobre: cálculo de probabilidad simple; cálculo de una probabilidad condicional y cálculo de una probabilidad conjunta. Al analizar las respuestas se detecta un gran número de errores en todas ellas, sobre todo en las preguntas referidas a probabilidad condicional y conjunta (Estrada y Díaz 2007).

Ortiz, Batanero y Contreras evalúan el conocimiento sobre la idea de juego equitativo de 167 profesores en formación de Educación Primaria. Para ello, analizan las soluciones dadas a dos problemas de respuesta abierta. Los resultados, a diferencia de los resultados obtenidos por Azcárate (1995), muestran que la gran mayoría de los futuros profesores tiene un conocimiento suficiente sobre la idea de juego equitativo y aplica estrategias, en su mayoría correctas, para decidir si un juego es o no equitativo. Los autores creen que esto se debe a que el problema que propuso Azcárate (1995) dificultó la tarea por falta de razonamiento combinatorio y no por falta de comprensión de la idea de juego equitativo (Ortiz, Batanero y Contreras 2012).

Mohamed evalúa el conocimiento común del contenido de 283 profesores en formación de Educación Primaria en relación a la idea de juego equitativo, a través de sus respuestas a un problema extraído de un libro de texto de educación primaria. Los resultados evidencian que la mayoría de los futuros profesores tienen un escaso conocimiento común del contenido en relación a la idea de juego equitativo, puesto que son incapaces de identificar y aplicar correctamente la idea de juego equitativo a la resolución de los problemas planteados. Dentro de los errores y dificultades que aparecen con mayor frecuencia se encuentran: el sesgo de la equiprobabilidad y de la falacia del jugador, la incorrecta realización de cálculos de probabilidad, la falta de capacidad combinatoria que les ha impedido determinar completamente el espacio muestral. Por último, cabe destacar que casi un $25 \%$ de los futuros profesores no contestó el problema planteado (Mohamed 2012).

Gómez evalúa el conocimiento común del contenido sobre probabilidad de acuerdo con los distintos significados de la probabilidad en profesores en formación de Educación Primaria, si bien los resultados son bastante alentadores, éstos indican un pobre razonamiento probabilístico y un predominio de las estrategias aritméticas (Gómez 2014).

Algunos autores analizan el conocimiento didáctico-matemático del profesorado de Educación Primaria para enseñar probabilidad, centrándose específicamente en la subcategoría de conocimiento común del contenido. Para ello, se analizaron las prácticas matemáticas de 93 profesores chilenos de Educación Primaria en activo a partir de un cuestionario compuesto por 7 ítems que evalúan aspectos parciales e iniciales de dicho conocimiento. Los resultados muestran un nivel de conocimientos muy insuficiente, con 4,75 puntos promedio de respuestas correctas sobre 14 .

Como es posible observar, la literatura reporta numerosos trabajos en torno a la comprensión de la probabilidad en el profesorado de primaria, sin embargo, los estudios centrados en el profesorado de secundaria son menores, más aún en Chile.

Por su parte, Díaz, Contreras, Batanero y Roa estudian los sesgos relacionados con el razonamiento probabilístico condicional de 196 sujetos, entre estudiantes del último año del grado de matemática y futuros profesores de secundaria en matemática en España. Los resultados muestran un predominio de la falacia del eje temporal, la condicional transpuesta, confusión entre probabilidad condicional y conjunta, falacia de las tasas base y concepciones incorrectas sobre la independencia (Díaz, Contreras, Batanero y Roa 2012). 
Bastias, Alvarado y Retamal indagan sobre el significado intuitivo de la probabilidad de 118 profesores de secundaria chilenos por medio de un instrumento con 8 ítems sobre la asignación intuitiva de probabilidades en contextos de contingencia nacional. Los resultados evidencian la diversidad de puntuaciones en los diferentes ítems, reflejando la existencia de sesgos de razonamiento, y evidenciando la débil preparación de estos profesores (Bastias, Alvarado y Retamal 2017).

Cardeñoso, Moreno, García-González, Jiménez-Fontana analizan la presencia del sesgo de equiprobabilidad en el razonamiento de 908 futuros profesores de Matemática y de Biología en Argentina. Para la recolección de los datos se analizaron las respuestas a 12 preguntas relacionados a la estimación de la probabilidad a un determinado fenómeno. Los resultados muestran que los profesores en formación usan desmedidamente la equiprobabilidad para justificar sus estimaciones probabilísticas; siendo significativamente mayor en quienes estudian Biología. Además, las dificultades se observan mayoritariamente en los contextos físico-natural y cotidiano (Cardeñoso, Moreno, García-González, Jiménez-Fontana 2017).

Inzunsa y Guzmán investigan sobre los conocimientos y comprensión del objeto matemático probabilidad que muestran profesores de secundaria mexicanos. Los resultados muestran que el concepto de probabilidad es un tema complicado para ellos. Además, observaron que lo relacionado con el razonamiento combinatorio bajo en la mayoría de los participantes del estudio, son que utilizan pocas representaciones como diagramas de árbol y de Venn (Inzunsa y Guzmán 2011).

Es claro que la enseñanza de la probabilidad, pasa por tener profesores entusiasmados por el tema y sobre todo bien preparados. Sin embargo, algunos profesores en activo y en formación pueden sentirse inseguros al enseñar la probabilidad por no haber recibido suficiente formación disciplinaria y didáctica sobre el tema. Esto se confirma en algunos estudios con profesores en diferentes países que, aún siendo conscientes de la importancia del tema para sus estudiantes indican diversas razones para reducir u omitir su enseñanza, como la falta de tiempo debido a lo apretado de los programas o su inseguridad para enseñarlo (Alpízar, Chavarría y Oviedo 2015). Para Aparicio y Bazán los docentes no imparten temas relacionados con probabilidad debido a la escasa formación entregada las instituciones formadoras, lo que hace que cuente con pocos recursos a la hora de dar sus clases y tienda a omitir el tema; acortarlo o, en el mejor de los casos, a presentarlo con una metodología inadecuada (Aparicio y Bazán 2006).

\section{METODOLOGÍA}

\subsection{DISEÑO DEL ESTUDIO}

Esta investigación es de naturaleza mixta (Hernández, Fernández, y Baptista 2010; PereiraPérez 2011) y utilizó una complementariedad metodológica (Aldana 2007; AngueraArgilaga 2004; Blanco y Pirela 2016), atendiendo a que se compararon las habilidades (conocimiento) asociadas a la alfabetización probabilística y el análisis de las narrativas de los profesores en activo y en formación, estos últimos de la carrera de Pedagogía en Matemática de una universidad pública del centro sur de Chile. En lo cuantitativo, se utilizaron de métodos descriptivos numéricos (porcentaje, promedio, desviación estándar) y en lo cualitativo se analizan las narrativas de los encuestados. 


\subsection{PARTICIPANTES}

Para efectos del estudio se realizó un muestreo no probabilístico de tipo intencionado o por conveniencia (Azorín y Sánchez-Crespo 1986). La muestra estuvo formada por un total de 45 sujetos, de los cuales 30 corresponden a profesores en formación pertenecientes a una universidad tradicional del centro sur de Chile, y 15 profesores en activo del sistema escolar que asisten a perfeccionamiento en la misma institución.

En la Tabla 3, se muestra la caracterización de los sujetos en estudio, se puede observar que no presentan diferencias estadísticamente significativas en la distribución de género (hombres y mujeres). Los profesores tienen una edad promedio de 32,3 años los que se han desempeñado en promedio 7,5 años en el sistema escolar cuya distribución es relativamente heterogénea (D.E=8,0 años) y asimétrica positiva (C.A=0,7), en general se desarrollado docencia en el sistema municipal (60\%). Por su parte los estudiantes FID presentan una edad promedio de 22,6 años, provienen mayoritariamente de colegios particulares subvencionados (67\%), no obstante, el 33\% realizó estudios en el sistema municipal.

Tabla 3. Estadísticas básicas de los profesores en formación y en activo considerados en el estudio

\begin{tabular}{|l|l|c|c|}
\hline \multicolumn{1}{|c|}{ Variable } & \multicolumn{1}{|c|}{ Categoría } & Profesores en formación & Profesores en activo \\
\hline Género & Femenino & $65 \%$ & $60 \%$ \\
\hline & Masculino & $35 \%$ & $40 \%$ \\
\hline Edad (años) & --- & $22,6(1,8)$ & $32,3(11,0)$ \\
\hline Años de Servicio & --- & & $7,5(8,0)$ \\
\hline Dependencia & Municipal & $33 \%$ & $60 \%$ \\
\hline & P. Subvencionado & $67 \%$ & $40 \%$ \\
\hline
\end{tabular}

\subsection{INSTRUMENTOS DE RECOLECCIÓN DE DATOS}

Para efectos del estudio se aplicó un instrumento de selección múltiple sobre alfabetización probabilística cuyos reactivos se adaptan y están en correspondencia con el modelo propuesto por Gal (Gal 2005) y las directrices de Educación Primaria y Secundaria chilenas. $\mathrm{El}$ análisis de fiabilidad arrojó un valor de a=0,78, indicando que el instrumento posee una consistencia interna adecuada para los efectos del estudio, en la Tabla 4, que se muestra a continuación se desglosan sus especificaciones. 
Tabla 4. Especificaciones del instrumento de conocimientos relacionados con alfabetización probabilística según Modelo de Gal (2005)

\begin{tabular}{|l|l|c|}
\hline \multicolumn{1}{|c|}{ Contenido } & \multicolumn{1}{|c|}{ Habilidad } & $\begin{array}{c}\text { Número de } \\
\text { Preguntas }\end{array}$ \\
\hline Aleatoriedad, secuencias aleatorias. & Identificar propiedades de secuencias aleatorias. & 2 \\
\hline Regla de Adición de Probabilidades & $\begin{array}{l}\text { Identificar intersección de eventos y exclusión. } \\
\text { Estrategias de resolución. }\end{array}$ & 2 \\
\hline $\begin{array}{l}\text { Interpretación de probabilidades } \\
\text { desde un enfoque frecuencial. }\end{array}$ & $\begin{array}{l}\text { Identificar variabilidad de las frecuencias de } \\
\text { resultados de experimentos aleatorios }\end{array}$ & 3 \\
\hline $\begin{array}{l}\text { Razonamiento combinatorio y y } \\
\text { cálculo de probabilidades }\end{array}$ & $\begin{array}{l}\text { Identificar razonamiento combinatorio y uso de } \\
\text { representaciones gráficas }\end{array}$ & 4 \\
\hline
\end{tabular}

\subsection{PROCEDIMIENTO DE ANÁLISIS.}

Los resultados se presentan mediante un análisis descriptivo numérico (porcentaje, promedio, desviación estándar), El procesamiento de la información fue realizado en software de fuente abierta, los datos cuantitativos fueron procesados en el paquete estadístico R 2.1.1 y la información cualitativa en el software Qualitative Data Analysis versión 4.0 (QDA).

\section{RESULTADOS}

Se presentan en cuatro secciones que están en estrecha relación con las habilidades de alfabetización estadística de los alumnos en Formación Inicial Docente (FID) y profesores en ejercicio del sistema escolar. Se discuten los argumentos que esgrimen los sujetos, en relación a la descodificación de las situaciones problemáticas entregadas en las cuales se han puesto en juego conceptos estadísticos de uso común.

\subsection{ALEATORIEDAD EN PROFESORES EN FORMACIÓN Y EN ACTIVO}

Uno de los conceptos ampliamente utilizados en estadística es el de aleatoriedad, entendiendo que la estadística está asociada a fenómenos estocásticos. En el sistema escolar chileno, la introducción de este concepto a nivel curricular se inicia desde los primeros cursos de Educación Primaria. Noción que se coloca en juego mediante dispositivos físicos como el lanzamiento de una o dos monedas y observar su respuesta (cara o sello), donde el estudiante debe responder a la interrogante: ¿se puede predecir previamente la disposición en que caerán las monedas?, es decir, es posible conocer a priori el resultado del experimento. 
Tabla 5. Porcentaje promedio de logro entre profesores en formación y en activo

\begin{tabular}{|l|c|c|}
\hline \multicolumn{1}{|c|}{ Contenido } & Profesores en formación & Profesores en activo \\
\hline $\begin{array}{l}\text { Identificar una sucesión aleatoria al lanzar } \\
\text { una moneda legal cinco veces. }\end{array}$ & 77,0 & 64,3 \\
\hline $\begin{array}{l}\text { Comparar dos sucesiones y decidir cuál es } \\
\text { aleatoria. }\end{array}$ & 7 & 0 \\
\hline
\end{tabular}

En coherencia con lo anterior, en la Tabla 5 se muestran los porcentajes de logro de los grupos en relación a identificar secuencias aleatorias, en particular del lanzamiento de una moneda cinco veces.

En la cual el 64,3\% de los profesores declaran que las sucesiones CCCSS, CSSCS, CSCSC y SCSSS son igualmente probables. Entregando argumentos como:

"Las cuatro sucesiones son igualmente probables al igual que cualquier otra sucesión posible, esto debido a que en probabilidades no existe un patrón que indique que situación es más factible que ocurra" (Profesor 1, Género Femenino).

Notar que este profesor hace un símil entre experimento aleatorio y probabilidad, al expresar que:

“...debido a que en probabilidades no existe un patrón que indique que situación es más factible que ocurra”.

Situación que a nivel de sistema escolar puede generar ruido en los alumnos. De manera similar el profesor 9 de género masculino argumenta que:

"La base del azar es la equiprobabilidad de ocurrencia de los sucesos, es decir, que todos los sucesos tienen la misma probabilidad de ocurrencia. (Profesor 9, Género Masculino).

No obstante, uno de los profesores explicita que:

"Es más probable que aparezca CCCSS por que el hecho de que la moneda este equilibrada indica una sucesión pareja" (Profesor 5, Género Femenino).

Llama la atención que el profesor 5, en su relato, hace relación de manera implícita a una suerte de patrón el cual no está asociado a una sucesión de carácter aleatoria, entendido que para que un fenómeno sea aleatorio no debería presentar una regularidad.

En relación al comparar la secuencia del lanzamiento de una moneda 40 veces por dos estudiantes y decidir cuál de ellas es aleatoria. Se tiene que el alumno 1 tiene 19 caras $(47,5 \%)$ y el alumno 2 obtiene 12 (30\%), dado que la moneda es legal -teóricamente hay una probabilidad del 0,5 de obtener cara o obtener sello, en términos porcentuales tenemos que el 50\%, son caras- de aquí el porcentaje de caras del alumno 1 es más cercano a este 
valor, esto nos podría hacer pensar que no hizo trampas, en cambio el alumno 2 lo más probable que si lo hizo. Escenario que no es observado por los profesores en activo, dado que no respondieron este reactivo y solamente un $7 \%$ de los estudiantes en formación entrega una respuesta correcta. $\mathrm{Al}$ respecto algunas argumentaciones son:

"No es fácil saber quién hizo o no el experimento, ya que si observamos el número de lanzamientos ambos son 40 y el número de caras y sellos se van alternando, por lo tanto, podría decir que ambos si pudieron haber realizado el experimento". (Profesor 1, Género Femenino).

"Considero que Juan no realizo el experimento porque en sus lanzamientos sigue un patrón CSSC... que la mayoría de las veces y sus resultados de los lanzamientos son muy parejos y muy largo la probabilidad de que esto suceda es muy baja” (Profesor 4, Género Masculino).

En cambio, los profesores en formación argumentan:

"No se puede saber, quien lo hizo y quien no" (Estudiante 13, Género Femenino) Y la gran mayoría comenta "No lo sé" o dejan en blanco el espacio de respuesta.

En general las argumentaciones dadas tanto por profesores en formación como en activo presentan argumentaciones similares y poco plausibles, dejando en evidencia que el concepto de aleatoriedad no es tratado con la importancia que tiene en probabilidad en las asignaturas de estadística en sus itinerarios formativos, potenciando las habilidades algorítmicas por sobre las conceptuales.

\subsection{CÁLCULO DE PROBABILIDAD POR PROFESORES EN FORMACIÓN Y EN ACTIVO}

En Chile, el estudio de la probabilidad se presenta desde primer año básico (6 años de edad) y de manera progresiva hasta finalizar la Educación Secundaria, enfatizando en la necesidad de desarrollar los conocimientos y habilidades necesarios para una adecuada comprensión de la probabilidad, abordando para ello conceptos básicos y distintas aplicaciones de las probabilidades que deben permitir establecer, de manera progresiva, conexiones entre las matemáticas y otros ámbitos del saber, así como con experiencias de la vida diaria. Así a partir de esta visión de la probabilidad como herramienta para el análisis de situaciones de vida cotidiana, es que el cálculo de probabilidades es abordado como tal en los últimos cursos de Educación Primaria, donde la probabilidad de un suceso (o evento) como la proporción del número de casos favorables al número de casos posibles, siempre que todos los casos sean igualmente posibles (Regla de Laplace). En la Tabla 6 se muestran los porcentajes de logro de los profesores en formación y en activo en relación al cálculo de probabilidades, en las que participan actividades tales como la probabilidad "conjunta", la unión de dos eventos y el cálculo de la probabilidad de un evento complementario. 
Tabla 6. Porcentaje de logro entre profesores en formación y en activo en el cálculo de probabilidades

\begin{tabular}{|l|c|c|}
\hline \multicolumn{1}{|c|}{ Contenido } & $\begin{array}{c}\text { Profesores en } \\
\text { formación }\end{array}$ & $\begin{array}{c}\text { Profesores en } \\
\text { activo }\end{array}$ \\
\hline Probabilidad que ocurra el evento A y el evento B. & 23,1 & 28,6 \\
\hline Probabilidad que ocurra el evento complementario & 61,5 & 64,2 \\
\hline Regla de la adición de probabilidades & 30,7 & 71,4 \\
\hline
\end{tabular}

De la Tabla 6 se observa que los profesores en activo presentan porcentajes de logro mayor que quienes se encuentran en formación, como la probabilidad que ocurra el evento complementario.

Algunos argumentos dados por los profesores en activo son:

"Donde me pide que una probabilidad la posibilidad disminuye por lo que hay que multiplicar y donde se piden por separado, la probabilidad aumenta hay que sumar. (Profesor 1, Género Femenino).

Una de las argumentaciones que realizan los estudiantes:

"Se pide que cumpla dos requisitos hay que multiplicar las probabilidades, como pueden ocurrir dos casos hay que sumar las probabilidades, la mitad son números pares y la otra mitad impares" (Estudiante 1. Género Femenino).

Dado que en general no entregan argumentaciones en relación a las interrogantes presentadas, por lo que se asume que argumentación es sinónimo de cálculo.

\subsection{INTERPRETACIÓN DE PROBABILIDADES DESDE UN ENFOQUE FRECUENCIAL DE PROFESORES EN FORMACION Y EN ACTIVO}

El enfoque frecuencial de la probabilidad, plantea la asignación de probabilidades a partir de la frecuencia relativa observada en un gran número de repeticiones, permitiendo estimar la probabilidad del suceso. Así, la "Ley de los Grandes Números", indica que la probabilidad de que la frecuencia relativa de un experimento repetido en las mismas condiciones se acerque a la probabilidad teórica del suceso, puede aproximarse suficientemente a 1, sin más que aumentar el número de pruebas. En la Tabla 7 se muestran los resultados obtenidos al descodificar información de tipo textual y gráfica en que interviene el objeto matemático probabilidad. En ella se observa que el 84,6\% de los futuros profesores descodifica correctamente un enunciado de tipo probabilístico, porcentaje de logro que disminuye a un $42,8 \%$ en el grupo de profesores en activo en el sistema escolar. 
Tabla 7. Porcentaje promedios de logro de los sujetos en estudio en relación a la descodificación de información del tipo probabilística

\begin{tabular}{|l|c|c|}
\hline \multicolumn{1}{|c|}{ Descodificación de información } & $\begin{array}{c}\text { Profesores en } \\
\text { formación }\end{array}$ & $\begin{array}{c}\text { Profesores en } \\
\text { activo }\end{array}$ \\
\hline Probabilística presente en un enunciado de tipo textual & 84,6 & 42,8 \\
\hline $\begin{array}{l}\text { Del comportamiento probabilístico del lanzamiento de una } \\
\text { moneda mediante un polígono de frecuencia simple. }\end{array}$ & 38,4 & 42,8 \\
\hline $\begin{array}{l}\text { Del comportamiento del lanzamiento de un dado mediante } \\
\text { un histograma. }\end{array}$ & 23 & 42,8 \\
\hline
\end{tabular}

Al respecto, algunas argumentaciones entregadas por los profesores del sistema escolar son:

"El 15\% significa, 15 de cada 100, si tomamos en cuenta que quien se aplica este medicamento son personas, entonces podemos decir que 15 de cada 100 personas han sufrido irritación en la piel". (Profesor 1, Género Femenino).

$15 \%$ de probabilidad de irritación da lo mismo el área cubierta con la crema es la reacción en cuento al organismo de cada persona estos efectos siempre se miden en cantidad de personas y se expresan en \% (Profesor 14, Género Masculino).

"Porque informa que de 100 personas solo 15 reaccionamos ese al $15 \%$ de $100 "$. (Profesor 6, Género Masculino).

De manera similar, algunas de las argumentaciones entregadas por los profesores en formación son:

"Por qué \% significa cada 100, entonces $15 \%$ sería 15 de cada 100". (Estudiante 1, Género Femenino).

"Se supone que hay un estudio de cómo afecta el medicamento a las personas". (Estudiante 5, Género Femenino).

"Para las personas que no manejan bien las probabilidades es más fácil leer 15 de cada 100 en vez de 15\%" (Estudiante 12, Género Masculino).

En relación a la descodificación de representaciones gráficas los profesores en activo del sistema escolar presentan porcentajes de logro mayor $(42,8 \%)$ en relación a los profesores en, resultados que coinciden con los hallazgos de Autores.

"La moneda estaba sesgada ya que la frecuencia nunca bajo de 0,6 en ninguno de los 30 lanzamientos y sus resultados son siempre bastantes parejos, fluctúan entre 0,6 y 1,0”. (Profesor 6, Género Masculino). 
"Si los datos fuesen constantes, sabríamos si era legal o estaba sesgada, pero al ser irregulares al principio y luego más constante es difícil determinar" (Profesor 11, Género Femenino).

"Pues nos los entrega un valor de los datos cuando están sesgados o no, solo arroja un valor" (Estudiante 6, Género Femenino).

"Por qué las probabilidades son bastante cercanas a 1" (Estudiante 9, Género Masculino).

La interpretación de resultados juega un rol fundamental al momento de pensar en la alfabetización probabilística, la cual en ambos grupos resulta deficitaria dado que entregan argumentos poco plausibles en relación a la situación problemática a la que son sometidos.

\subsection{RAZONAMIENTO COMBINATORIO Y CÁLCULO DE PROBABILIDADES DE PROFESORES EN FORMACIÓN Y EN ACTIVO}

A nivel de Educación Primaria se introducen ideas tendientes a conocer, a priori, la cardinalidad de un espacio muestral con la introducción de reglas de conteo. En particular, el principio multiplicativo, se ubica en las actuales orientaciones curriculares chilenas en el octavo curso de primaria, definiéndola en términos simples como "una técnica de conteo sencilla que permite determinar el número de elementos de un espacio muestral" (Catalán, Pérez, Prieto y Rupin 2016). Por tanto, es un tema que el profesorado que se desempeñe en este nivel debe conocer y comprender.

En la Tabla 8 se observan los porcentajes de éxito que alcanzan los profesores en activo y en formación respecto a esta temática. En ella, ambos grupos presentan porcentajes de logro bajos, del orden del 7,6\% para los profesores en formación y 7,1\% para los profesores en activo. En cambio, el 57,1\% de los profesores presenta la habilidad de tomar decisiones en base a la comparación de probabilidades de decidir si era más probable de obtener "un cinco y un seis" u obtener "seis y seis" al lanzar dos dados legales, porcentaje que disminuye a $38,5 \%$ en los profesores en formación.

Tabla 8. Porcentajes de logro en relación a situaciones problemáticas donde intervienen la regla multiplicativa, probabilidad condiciones y comparación de probabilidades

\begin{tabular}{|l|c|c|}
\hline Descodificación de información & $\begin{array}{c}\text { Profesores en } \\
\text { formación }\end{array}$ & $\begin{array}{c}\text { Profesores en } \\
\text { activo }\end{array}$ \\
\hline Regla multiplicativa & 7,6 & 7,1 \\
\hline Comparación de probabilidades & 38,5 & 57,1 \\
\hline Juego equitativo & 23 & 35,7 \\
\hline Probabilidad Condicional & 30,7 & 57,1 \\
\hline
\end{tabular}


Al comparar probabilidades relacionadas con el lanzamiento simultáneo de dos dados, los profesores argumentan que:

"Es más probable obtener un 5 y un 6 ya que el primer dado caer en 5 o 6 al igual que el segundo dado tienen dos opciones. Mientras que para que sea 6 y 6 ambos dados solo tienen una opción" (Profesor 3, Género Masculino).

"Es más probable que salgan 2 números distintos a que salgan 2 iguales (son 5 números distintos, 6 es solo 1)" (Profesor 12, Género Femenino).

"En ambas existe la misma probabilidad de 36 posibles combinaciones la probabilidad es 1/36" (Profesor 8, Masculino).

En cambio, los estudiantes para profesores presentan argumentos como:

"Son los valores que considero que serían los más esperados ya que obtener seis en ambos dados es caso extremo y las probabilidades se distribuyen en los valores cercanos a la media" (Estudiante 4, Masculino).

"Por qué hay una posibilidad entre 36 de obtener un seis en los dos dados, pero obtener 5 y seis podrían ser distinto orden, 5 y 6 ó 6 y 5, y ahí habría 2/36 de probabilidad" (Estudiante 8, Género Femenino).

En el caso de probabilidad condicional los profesores del sistema argumentan:

"La probabilidad de sacar una ficha de dos caras rojas es $1 / 2$ y la probabilidad de sacar una ficha con cara roja y azul es $1 / 2$. La probabilidad de que la ficha tenga la otra cara roja es igual, a la que tenga la cara azul" (Profesor 1, Género Femenino).

"Si la primera ficha que se extrajo tiene una cara roja eso implica que la ficha que tiene rojo y azul solo podría salir en la segunda extracción solo en 1 de 4 casos por eso es más probable que sea la ficha con ambas caras rojas" (Profesor 9, Género Masculino).

Y, por su parte, los estudiantes para profesores:

"Hay solo dos opciones, roja o azul, son igual de probables" (Estudiante 1, Género Femenino).

"Ya que la de ambos colores está condicionada a salir con probabilidad 1/4" (Estudiante 10, Género Masculino).

A pesar de tener una formación algorítmica en general ambos grupos en estudio presentan porcentajes de logro bajos en relación a dar respuesta a situaciones problemáticas siendo un problema transversal la argumentación 


\section{DISCUSIÓN Y CONCLUSIÓN}

La incorporación de la estadística y la probabilidad en las directrices curriculares chilenas de Educación Primaria, y con ellos su tratamiento más amplio y complejo en Educación Secundaria, plantea diversos desafíos para los profesores en formación y activo del sistema escolar en general. Lo que tensiona cambios importantes en los itinerarios formativos, quienes son los responsables de transmitir a sus futuros estudiantes contenidos y generar las habilidades que exige este nuevo eje.

Dentro de los contenidos que deben ser entregados por los profesores en activo y en formación está el objeto estadístico probabilidad, en el cual se encuentra de manera implícita la idea de aleatoriedad y azar, conceptos con una gran carga historia y de carácter polisémico como lo han hecho saber diferentes autores (Batanero y Serrano 1995; Cuche 2002; Zabell 1992). Teniendo presente que los futuros profesores y en activo deben ser "capaz de conducir el aprendizaje de los estudiantes en la introducción de conceptos relativos a probabilidades, utilizando para esto situaciones lúdicas y cotidianas que ilustren como cuantificar el azar, considerando las dificultades que esto conlleva en cada nivel" (Ministerio de Educación 2012b).

Pese a esta importancia del tema, múltiples trabajos muestran las dificultades que tienen los profesores (en formación y en activo) de Educación Primaria (e.g., Azcárate 1995; Begg y Edward 1999; Estrada y Díaz 2007; Ortiz et al. 2012; Gómez 2014; Mohamed 2012) y de Educación Secundaria (e.g, Bastias et al. 2017; Cardeñoso et al. 2017; Díaz et al. 2012; Inzunsa y Guzmán 2011).

En general, en muestro estudio, los profesores en activo presentan porcentajes de logros levemente superiores a los profesores en formación, ante situaciones problemáticas donde deben decidir de manera intuitiva si una sucesión es o no aleatoria, cabe hacer notar que en promedio los profesores en activo tienen 7,5 años de ejercicio docente logran en promedio un $70 \%$ de logro y no un $100 \%$ como se podría haber pensando dado que los libros de texto entregados por el Ministerio de Educación presenta actividades de este tipo y en algunos presenta comentarios tendientes a explicar si una sucesión es o no aleatoria, evidenciando una vez más que los libros de texto no son utilizados en el sistema escolar.

La argumentación es una de las habilidades que deben presentar los profesores en formación y activo del sistema escolar, más aún en un eje cuyo impronta es el trabajo con datos reales, teniendo presente como menciona Moore la estadística es la ciencia de los datos y a nivel de sistema escolar su principal objetivo es alfabetizar estadísticamente y probabilísticamente a los estudiantes, futuros ciudadanos del siglo XXI en un mundo cada día más globalizado Donde circula gran cantidad de información de tipo cultural, política, económica, educativa o social en la cual la incertidumbre se encuentra presente ( $\mathrm{Gal} 2005$ ).

En este contexto, los grupos en estudio profesores en formación y activo en general esgrimen argumentaciones poco plausibles a la hora de explicar por qué un fenómeno aleatorio puede ser o no considerado aleatorio, al hacer uso de la regla aditiva, o cálculo de probabilidades de sucesos complementarios.

Lo anterior, genera una tensión entre las habilidades y conocimientos que deben adquirir durante su trayectoria formativa las que deben colocar en juego durante su desempeño profesional; más aún ahora a nivel chileno con la incorporación de la nueva carrera docente (Ley 20903), dado que nadie enseña lo que no sabe, generando baches en la formación de los estudiantes y por consiguiente invisibilizado la potencialidad del eje en la formación. 
Finalmente, el presente estudio, proporciona evidencias sobre cómo los profesores en activo y en formación visualizan conceptos que están de manera explícita en el objeto probabilidad y también aporta información sobre habilidades que deben ser potenciadas en los itinerarios formativos en estos grupos en estudio, antecedentes que servirían de insumo a nivel país de algunas casas de estudio cuando a las nuevas políticas de renovación curricular en el marco de las competencias profesionales atendiendo a los nuevos lineamientos curriculares impulsados por el Ministerio de Educación chileno.

\section{REFERENCIAS BIBLIOGRÁFICAS}

Aldana, G. M. (2007). Complementariedad metodológica en la investigación social. Una propuesta de integración. Pedagogía y Saberes, 26, 51-56. DOI: 10.17227/01212494.26pys51.56

Alpízar, M., Chavarría, L. y Oviedo, K. (2015). Percepción de un grupo de docentes de I y II ciclo de educación general básica de escuelas públicas de Heredia sobre los temas de estadística y probabilidad. Actualidades Investigativas en Educación, 15 (1), 1-23. DOI: 10.15517/aie. v15i1.17728

Alsina, Á (2012). La estadística y la probabilidad en Educación Infantil: conocimientos disciplinares, didácticos y experienciales. Didácticas Específicas, 7, 4-22. Recuperado el 15 de octubre de 2017 desde https://revistas.uam.es/didacticasespecificas/article/view/7700/7976

Anguera-Argilaga, M. T. (2004). Posición de la metodología observacional en el debate entre las opciones metodológicas cualitativa y cuantitativa. Enfrentamiento, complementariedad, integración?. Psicologia em Revista, 10 (15), 13-27.

Aparicio, A. y Bazán, J. L. (2006). Actitud y rendimiento en Estadística en profesores peruanos. En G. Martínez (Ed.), Acta Latinoamericana de Matemática Educativa 19 (pp. 644-650). México: CLAME.

Azcárate, P. (1995). El conocimiento profesional de los profesores sobre nociones de aleatoriedad y probabilidad. Su estudio en el caso de la Educación Primaria (Tesis doctoral). Universidad de Cádiz, España.

Azorín, F. y Sánchez-Crespo, J. (1986). Métodos y Aplicaciones del Muestreo. Madrid: Alianza.

Ball, D. L., Lubienski, S. T. y Mewborn, D. S. (2001). Research on teaching mathematics: The unsolved problem of teachers` mathematical knowledge. En V. Richardson (Ed.), Handbook of Research on Teaching (pp. 433-456). Washington: American Educational Research Association.

Batanero, C. (2001). Didáctica de la Estadística. Granada: Grupo de Investigación en Educación Estadística.

Batanero, C. (2005). Significados de la probabilidad en la educación secundaria. Revista Latinoamericana de Investigación en Matemática Educativa, 8 (3), 247-264. Recuperado el 12 de octubre de 2017 desde http://www.redalyc.org/articulo.oa?id=33508302

Batanero, C. (2006). Razonamiento probabilístico en la vida cotidiana: Un desafío educativo. Investigación en el aula de matemáticas. Estadística y Azar (pp. 1-17). Granada: Sociedad Andaluza de Educación Matemática Thales.

Batanero, C., Godino, J. D. y Cañizares, M. J. (2005). Simulation as a tool to train Pre-service School Teachers. En J. Addler (Ed.), Proceedings of ICMI First African Regional Conference (pp. 1-8). Johannesburgo: International Commission on Mathematical Instruction.

Batanero, C., Ortiz, J. J. y Serrano, L. (2007). Investigación en didáctica de la probabilidad. UNO. Didáctica de las Matemáticas, 44, 7-16.

Batanero, C. y Serrano, L. (1995). Aleatoriedad, sus significados e implicaciones educativas. UNO. Didáctica de las Matemáticas, 5, 15-28.

Bastias, H. Alvarado, H. y Retamal, L. (2017). Explorando el significado intuitivo de probabilidad 
en profesores de matemática. En J. M. Contreras, P. Arteaga, G. R. Cañadas, M. M. Gea, B. Giacomone y M. M. López-Martín (Eds.), Actas del Segundo Congreso International Virtual sobre el Enfoque Ontosemiótico del Conocimiento y la Instrucción Matemáticos (pp. 1-10). Granada: Universidad de Granada. Recuperado el 15 de octubre de 2017 desde http:// enfoqueontosemiotico.ugr.es/civeos/bastias.pdf

Begg, A. y Edwards, R. (1999). Teachers' ideas about teaching statistics. En AARE y NZARE (Eds.), Proceedings of the 1999 combined conference of the Australian Association for Research in Education and the New Zealand Association for Research in Education (pp. 1-9). Melbourne: AARE \& NZARE.

Ben-Zvi, D. y Garfield, J. (2004). Statistical literacy, reasoning, and thinking: goals, definitions, and challenges. En J. Garfield y D. Ben-Zvi (Eds.), The challenge of developing statistical literacy, reasoning and thinking (pp. 3-15). Netherlands: Springer.

Blanco, N. y Pirela, J. (2016). La complementariedad metodológica: estrategia de integración de enfoques en la investigación social. Espacios Públicos, 18 (45), 97-111. Recuperado el 10 de octubre de 2017 desde http://politicas.uaemex.mx/espaciospublicos/images/stories/fruit/PDF/ Rev_45/05-La_complementariedad.pdf

Cardeñoso, J. M., Moreno, A., García-González, E. y Jiménez-Fontana, R. (2017). El sesgo de equiprobabilidad como dificultad para comprender la incertidumbre en futuros docentes argentinos. Avances de Investigación en Educación Matemática, 11, 145-166. Recuperado el 2 de octubre de 2017 desde www.aiem.es/index.php/aiem/article/view/185/121

Catalán, D., Pérez, B., Prieto, C. y Rupin, P. (2016). Texto del estudiante. Matemática $8{ }^{\circ}$ básico. Santiago: SM.

Cuche, D. (2002). La noción de cultura en las ciencias sociales. Buenos Aires: Nueva Visión.

Del Pino, G. y Estrella, S. (2012). Educación estadística: relaciones con la matemática. Pensamiento Educativo. Revista de Investigación Educacional Latinoamericana, 49 (1), 53-64. DOI: 10.7764/ PEL.49.1.2012.5

Díaz, C., Contreras, J., Batanero, C. y Roa, R. (2012). Evaluación de sesgos en el razonamiento sobre probabilidad condicional en futuros profesores de Educación Secundaria. BOLEMA. Boletim de Educação Matemática, 26 (44), 1207-1225. Recuperado el 25 de noviembre de 2017 desde http://www.scielo.br/pdf/bolema/v26n44/06.pdf

Estrada, A. y Díaz, C. (2007). Errores en el cálculo de probabilidades en tablas de doble entrada en profesores en formación. UNO. Didáctica de las Matemáticas, 44, 48-58.

Estrella, S., Olfos, R. y Mena-Lorca, A. (2015). Pedagogical content knowledge of statistics among primary school teachers. Educação e Pesquisa, 41 (2), 477-493. Recuperado el 25 de noviembre de 2017 desde http://www.scielo.br/pdf/ep/v41n2/1517-9702-ep-41-2-0477.pdf

Even, R. y Ball, D. L. (2009). Setting the stage for the ICMI study on the professional education and development of teachers of mathematics. En R. Even y D. L. Ball (Eds.), The Professional Education and Development of Teachers of Mathematics (pp. 1-9). Boston: Springer.

Franklin, C. y Garfield, J. (2006). The guidelines for assessment and instruction in statistics education (GAISE) project: developing statistics education guidelines for pre K-12 and college courses. En G. Burrill (Ed.), Thinking and reasoning about data and chance: sixty-eighth NCTM Yearbook (pp. 345-375). Reston: NCTM.

Gal, I. (2002). Adult's statistical literacy: Meaning, components, responsibilities. International Statistical Review, 70 (1), 1-25.

Gal, I. (2005). Towards "Probability Literacy" for all Citizens: Building Blocks and Instructional Dilemmas. En G. Jones (Ed.), Exploring probability in school: Challenges for teaching and learning (pp. 39-63). Nueva York: Springer.

Gimeno, J. (1999). La educación que tenemos, la educación que queremos. En F. Imbernón (Ed.), La educación en el siglo XXI. Los retos del futuro inmediato (pp. 29-52). Barcelona: Grao.

Gómez, E. (2014). Evaluación y desarrollo del conocimiento matemático para la enseñanza de la 
probabilidad en futuros profesores de Educación Primaria (Tesis Doctoral). Universidad de Granada, España.

Hernández, R., Fernández, C. y Baptista, P. (2010). Metodología de la investigación (5ta Edición). México: McGraw Hill.

Inzunsa, S. y Guzmán, M. (2011). Comprensión que muestran profesores de secundaria acerca de los conceptos de probabilidad: un estudio exploratorio. Educación Matemática, 23 (1), 63-95. Recuperado el 20 de octubre de 2017 desde http://somidem.com.mx/descargas/Vol23-1.pdf

Konold. C. (1991). Understanding students' beliefs about probability. En E. von Glasersfeld (Ed.), Radical constructivism in Mathematics Education (pp. 139-156). Dordrecht: Kluwer.

Laplace, P. S. (1986). Ensayo filosófico sobre las probabilidades. Madrid: Alianza (trabajo original publicado en 1825).

Lecoutre, M. P. (1992). Cognitive Models and Problem spaces in "Purely Random" Situations. Educational Studies in Mathematics, 23 (6), 557-568.

Lecoutre, M. P. y Durand, J. L. (1988). Jugéments probabilistes et modéles cognitifs: étude d' une situation aléatoire. Educational Studies in Mathematics, 19 (3), 357-368.

Liljedahl, P. (2009). Components of mathematics teacher training. En R. Even y D. L. Ball (Eds.), The Professional Education and Development of Teachers of Mathematics (pp. 25-33). Boston: Springer.

Ministerio de Educación (2012a). Matemática educación básica. Bases curriculares. Santiago: Unidad de Currículum y Evaluación.

Ministerio de Educación (2012b). Estándares orientadores para egresados de carreras de Pedagogía en Educación Básica. Estándares pedagógicos y disciplinarios. Santiago: LOM Ediciones Ltda.

Ministerio de Educación (2012c). Estándares orientadores para carreras de pedagogía en Educación Media. Estándares pedagógicos y disciplinarios. Santiago: LOM Ediciones Ltda.

Mohamed, N. (2012). Evaluación del conocimiento de los futuros profesores de educación primaria sobre probabilidad (Tesis Doctoral). Universidad de Granada, España.

Nilsson, P. y Li, J. (2015). Teaching and Learning of Probability. En S. J. Cho (Ed.), The Proceedings of the 12th International Congress on Mathematical Education (pp. 437-442). Switzerland: Springer International Publishing.

Ortiz, J. J., Batanero, C. y Contreras, C. (2012). Conocimiento de profesores en formación sobre la idea de juego equitativo. Revista Latinoamericana de Investigación en Matemática Educativa, 15 (1), 63-91. Recuperado el 20 de octubre de 2017 desde http://www.scielo.org.mx/pdf/relime/ v15n1/v15n1a4.pdf

Pereira-Pérez, Z. (2011). Los diseños de método mixto en la investigación en educación: una experiencia concreta. Revista Electrónica Educare, 15 (1), 15-29. Recuperado el 7 de octubre de 2017 desde www.redalyc.org/pdf/1941/194118804003.pdf

Sánchez, E. (2009). La probabilidad en el programa de estudio de matemáticas de la secundaria en México. Educación Matemática, 21 (2) 39-77. Recuperado el 7 de octubre de 2017 desde http:// www.scielo.org.mx/pdf/ed/v21n2/v21n2a3.pdf

Serradó, A., Azcárate, P. y Cardeñoso, J. M. (2006). Analyzing teacher resistance to teaching probability in compulsory education. En A. Rossman y B. Chance (Eds.), Proceedings of the Seventh International Conference on Teaching Statistics (pp. 1-6). Salvador de Bahía: International Association for Statistical Education e International Statistical Institute.

Tversky, A. y Kahneman, D. (1982). On the psychology of prediction. En D. Kahneman, P. Slovic y A. Tversky (Eds.), Judgement under uncertainty: Heuristics and biases (pp. 69-83). Cambridge: Cambridge University Press.

Zabell, S. L. (1992). The quest for randomness and its statistical applications. En F. Gordon y S. Gordon (Eds.), Statistics for the XXI Century (pp. 139-166). Washington: The Mathematical Association of America. 